\title{
Efeitos em preço do compartilhamento de voos de companhias aéreas como reação à presença de um competidor de baixo custo
}

\author{
Wellington Carlos Costa ${ }^{1}$ e Alessandro Vinicius Marques de Oliveira²
}

\begin{abstract}
Resumo: Este trabalho analisa os efeitos concorrenciais de um tipo de aliança estratégica que vem recebendo maior atenção por parte das companhias aéreas: o acordo de compartilhamento de voos (Codesharing). Busca avaliar os efeitos em preços gerados com a implementação do codesharing quando efetuado entre duas grandes companhias aéreas incumbentes frente à entrada de um competidor de operação de custo baixo (Low Cost Carrier), tema ainda não explorado na literatura. Para isso, foi desenvolvida uma modelagem econométrica com emprego de estimador de efeitos fixos para aplicação em um estudo de caso evidenciado no início dos anos 2000, o "Codeshare Varig-TAM". O modelo investiga os efeitos no preço das passagens das empresas incumbentes após entrada do competidor Low Cost. Os resultados indicam reduções do preço médio em todos os casos. Para condições em que não existia o codeshare implementado, a redução de preços era 12,4\%. Por outro lado, quando o codeshare já estava em vigor, a redução foi apenas $8,3 \%$. Estes resultados permitem inferir que o codeshare, pode ser uma ferramenta estratégica das grandes empresas para suavizar a ação de redução de preços (e perdas de lucratividade) quando competindo diretamente com Low Cost Carriers.
\end{abstract}

Palavras-chave: transporte aéreo, compartilhamento de voo.

\begin{abstract}
This paper aims to study the competitive effects of a type of strategic alliance that has received much more attention from airlines: the codeshare agreement. The intention is to evaluate the price effects caused by the implementation of codeshare when established between incumbent carriers facing the entrance of a new competitor Low Cost Carrier, a subject not yet addressed in the literature. For this, we developed an econometric model using fixed-effects estimator for application in a case study evidenced in the early 2000s, known as "Codeshare Varig-TAM". The model analyses the effects on the price of the air tickets due to the entrance of a newcomer Low Cost Carrier. The results indicate reductions on the average price in all cases. In conditions where the codeshare was not implemented, the price reduction was $12.4 \%$. On the other hand, when the codeshare already established, the reduction was only $8.3 \%$. These findings allow us to conclude that the codeshare can be a strategic tool for dominant airlines to mitigate the action of price reduction (and impacts on profitability) when competing directly against Low Cost Carriers.
\end{abstract}

Keywords: codeshare, low cost carrier.

\section{INTRODUÇÃO}

O objetivo principal deste trabalho é avaliar os efeitos no preço das passagens com a incorporação de estratégias competitivas por parte das companhias incumbentes através da implementação de um acordo de compartilhamento de voos (codeshare), investiga-se o impacto do codeshare entre grandes companhias que necessitam reagir à entrada de um novo competidor de operação de baixo custo (Low Cost Carrier). Para isso, desenvolveu-se de uma modelagem econométrica com controle de efeitos fixos no nível de rota, empresa e tempo baseada na aplicação de um estudo de caso ocorrido entre os anos de 2003 e 2004 denominado nesta pesquisa como "Codeshare Varig-TAM".

Há duas décadas, o setor de transporte aéreo no Brasil iniciava um processo de desregulação, promovido pelo então órgão regulador, o Departamento de Aviação Civil (DAC). Passados vinte anos, constata-se que as medidas liberalizantes adotadas gradualmente, resultaram em impactos positivos para o transporte aéreo no Brasil (Oliveira et

\footnotetext{
1 Wellington Carlos Costa, Departamento de Transporte Aéreo, ITA. (wellington.sjc@gmail.com)

${ }_{2}$ Alessandro Vinicius Marques de Oliveira, Departamento de Transporte Aéreo, ITA. (a.v.m.oliveira@gmail.com)

Manuscrito recebido em 01/07/2014 e aprovado para publicação em 25/11/2014.

Este artigo é parte de TRANSPORTES v. 23, n. 1, 2015. ISSN: 2237-1346 (online). DOI: 10.14295/transportes.v23i1.814
}

al, 2011). Um dos fenômenos mais marcantes do setor aéreo no Brasil no início dos anos 2000 foi a popularização do modal aéreo, especialmente com a entrada da Gol Linhas Aéreas Inteligentes, em 2001, se tornando a primeira companhia aérea do Brasil a aplicar o modelo de negócio denominado Low Cost.

De acordo com Oliveira e Huse (2009), a política de desregulamentação visava reduzir barreiras de entrada para fomentar o acesso de novos competidores no mercado. Assim, a entrada da Gol foi um evento extremamente bem sucedido, pois a companhia aérea conseguiu introduzir rapidamente suas operações em todo o território nacional oferecendo passagens aéreas a preços mais acessíveis. Outro fator que colaborou com o ritmo de expansão da Gol foi a fragilidade financeira dos principais operadores daquela época, além da saída de Transbrasil e Vasp, que deixaram de operar em 2001 e 2004, respectivamente. Com isso, a Gol alcançou quase $30 \%$ de participação de mercado em menos de quatro anos de operação devido à significativa redução de ofertas de voos por parte dos concorrentes, facilitando ainda mais o acesso da novata Gol nos aeroportos mais disputados.

Deste modo, em poucos anos a Gol acabou colocando-se como parte de um arranjo de mercado mais complexo junto a Varig e TAM, as duas maiores empresas naquele período, que constituíram um acordo de compartilhamento de voos (codeshare) a fim de fortalecer ambas as companhias em busca de sinergias e por fim, uma eventual fusão. 
Este trabalho investiga uma estrutura de mercado que uniu um codeshare entre duas das maiores empresas aéreas nacionais e um entrante de modelo low cost na primeira metade da década passada. Propõem-se uma discussão sobre distintas estratégias competitivas para responder ao movimento de entrada de novos competidores, em especial as Low Cost Carriers, que tendem a oferecer passagens aéreas a preços abaixo da média praticada no setor. As principais estratégias registradas na literatura acadêmica para o mercado aéreo são: i) guerra tarifária (price war), ii) cisão de negócios (spin off), e, por fim, acordos comerciais de alianças ou compartilhamento de voos.

Segundo Costa (2010), empresas de diferentes segmentos econômicos vem buscando novas estratégicas para expandir seus negócios ou defender sua posição frente aos competidores, e muitas empresas aéreas tem optado por unir de esforços com parceiros ou até concorrentes, com destaque para alianças, acordos comerciais, fusões e aquisições, devido ao dinamismo e a franca evolução do setor aéreo nos últimos anos. Estratégias deste gênero são fartamente encontradas no mercado brasileiro em tempos recente: a Gol adquiriu duas competidoras diretas, a Varig em 2007 e a Webjet em 2011; a TAM adquiriu a operadora regional Pantanal em 2009 e depois se fundiu com a companhia chilena LAN Airlines em 2010 se tornando o maior grupo aéreo na América Latina; por fim, a fusão entre Azul e TRIP no ano de 2012 constitui a maior empresa aérea regional do país com mais de 100 destinos servidos.

De modo geral, a formação de alianças estratégicas no transporte aéreo passa por uma fase inicial em que se objetiva primordialmente o aumento de receita, depois ambas as empresas atuam conjuntamente na redução dos custos, até que se forma uma condição onde dificilmente a aliança será descontinuada. Assim, pode-se considerar que uma fusão ou aquisição entre as companhias seria uma evolução natural do processo (Doganis, 2001). A proposito, para o caso estudado, Varig e TAM sinalizaram que além da constituição do acordo de codeshare existia uma intenção de fusão das empresas.

No início do ano de 2003, Varig e TAM assinaram um protocolo de intenções para reestruturar o setor aéreo no país, eliminar o excesso de sobreposição de oferta de voos e consequentemente reduzir as perdas financeiras das companhias aéreas. Esta manifestação foi oficializada através do codeshare Varig-TAM, que contemplava o compartilhamento recíproco de voos, abrangendo, sobretudo as rotas sobrepostas. Por fim, este acordo visava uma futura fusão para constituir uma nova empresa de capital aberto e controle privado.

Para Gatignon et al (1997), que analisa a efetividade das reações estratégicas após a introdução de novos produtos pelos competidores, idealmente, a reação precisa ser implementada rapidamente, e identificou-se que a estratégia de redução de preço apesar de ser a mais explorada, não está relacionadas com o sucesso no combate ao novo entrante em longo prazo, pois não é uma estratégia sustentável. E sugere que a exploração de vantagens competitivas de outras naturezas, poderia ser mais eficiente, como a diferenciação de produto através de características inovadoras que destacam o produto ou serviço ofertado.

Assim, abre-se uma discussão sobre a eficiência das distintas estratégias competitivas, especialmente as alianças estratégicas, que destacamos nesta pesquisa através do estudo de caso do codeshare entre Varig e TAM, constituído pouco tempo depois da entrada do novo competidor (Gol) no mercado.

O artigo está dividido da seguinte forma: a Seção 2 apresenta uma revisão da literatura acadêmica; a Seção 3 expõe o estudo de caso a ser explorado neste trabalho; a Seção 4 apresenta a metodologia do trabalho e a modelagem econométrica; a Seção 5 apresenta os resultados e alguns comentários. Por fim, expressasse as principais conclusões obtidas.

\section{REVISÃO DA LITERATURA}

\subsection{Companhias Aéreas de Baixo Custo e Estratégias de Resposta das Empresas Incumbentes Frente aos Novos Entrantes}

Nas últimas décadas o modelo de negócio denominado Low Cost Carrier tem sido amplamente utilizado na indústria de transporte aéreo e abordado na literatura acadêmica. Em modo geral, a estratégia consiste em oferecer preços básicos extremamente competitivos e abaixo da média do setor, através de uma significativa redução da estrutura de custos. Para isso, faz-se necessária uma exemplar gestão de custos, como a utilização de frota padronizada, ausência de serviço de bordo, operação em aeroportos secundários, venda de passagem pela internet, entre outros (Hüschelrath e Müller, 2011). Este modelo de negócio recebeu grande destaque devido ao relevante estímulo de demanda advindo da redução do preço das passagens, potencializando a inclusão de novos passageiros no modal.

Predominantemente, os estudos acadêmicos investigam empiricamente fatores determinantes da entrada das Low Cost Carriers (Oliveira, 2008), os efeitos nos preços após a entrada de Low Cost Carriers (Wu, 2011). Considerando a expansão das Low Cost Carriers, as empresas incumbentes tem reagido com distintas estratégias para bloquear ou mitigar a ascenssão destes novos entrantes. As principais estratégias registradas de resposta a novos competidores na literatura acadêmica para o transporte aéreo, são três, a saber: i) redução no preço (price war), ii) cisão de negócios (spin off), e, por fim iii) acordos comerciais para alianças ou compartilhamento de voos (codeshare).

\subsection{Redução dos Preços (Price War)}

Apesar de ser uma das estratégias mais exploradas como resposta à entrada de Low Cost Carriers, a redução de preços não está necessariamente relacionada com a obtenção de sucesso no combate ao novo entrante em distintos setores (aviação, telecomunicações, medicamentos, entre outros), pois pode ser considerada uma estratégia simples para empresas incumbentes que não são capazes de aplicar vantagens competitivas de outras naturezas, como: inovação, diferenciação do produto ou ações de marketing (Gatignon et al, 1997).

De acordo com Windle e Dresner (1999), após a entrada de um competidor de baixo custo, as empresas incumbentes tendem a adotar estratégias de redução significativa do preço médio nas rotas em competição, mas não conseguem repassar o aumento nos preços para rotas menos competitivas a fim de compensar guerras tarifárias nas rotas em competição, pois teriam o risco de registrar uma retração na demanda ou estimular novos entrantes que buscam rotas com melhores margens de lucro. Assim a guerra tarifária 


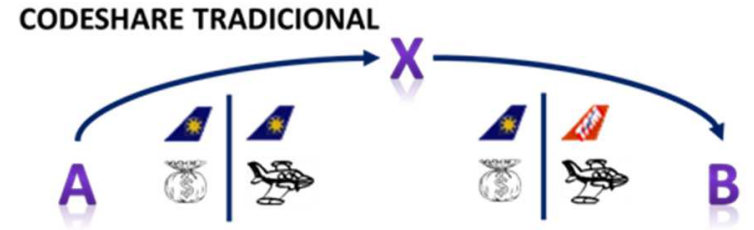

Marketing Airline

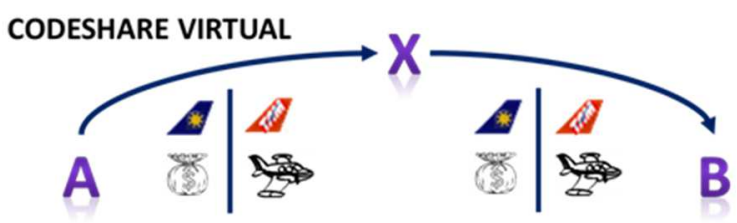

Operating Airline

Figura 1. Principais tipos de codeshare (Ito e Lee, 2007)

conduziria a um expressivo desequilíbrio de receita para a companhia aérea. Todavia, Tan (2011) que também analisa as resposta em preço relata um aumento dos preços para maximizar a exploração de serviços para o segmento de clientes cativos que identificam vantagens na diferenciação de produto disponibilizada e são fiéis à marca da companhia incumbente. Existiriam indícios de que a diferenciação de produto pode suavizar a necessidade de uma agressiva resposta em preços para bloquear um operador de baixo custo.

Predominantemente, as incumbentes reagem em preço somente após a entrada real, possivelmente devido à frágil situação financeira do setor nos últimos anos, o que inviabiliza uma reação antecipada. Esse fato foi analisado por Huse e Oliveira (2012), tendo sido apontadas evidências de falta de reações concorrenciais muito antecipadas. No entanto, outros estudos apontam para a existência de reações antecipadas. De acordo com Goolsbee e Syverson (2008), há casos em que os incumbentes podem praticar redução de preço mediante uma entrada potencial, baseado na probabilidade de entrada da Southwest Airlines em determinadas rotas. É possível identificar que algumas empresas incumbentes destas rotas potenciais podem reagir com redução de preços de modo significativo a partir de três meses de antecedência à entrada do operador de baixo custo na rota em questão.

\subsection{Cisão de Negócios (Spin off)}

Outra importante estratégia utilizada para combater a expansão de Low Cost Carriers nos Estados Unidos e Europa é denominada spin off, que consiste na criação de uma subsidiaria a partir da necessidade de explorar o mesmo mercado equivalente, praticando porém, outro modelo de negócio com produtos ou serviços diferentes.

Entretanto, esta estratégia não se mostrou eficiente nos casos analisados por Morrell (2005) devido à grande diferença na estrutura de custos entre as empresas incumbentes e as empresas genuinamente Low Cost. Mesmo assim, é possível identificar alguns raros casos bem sucedidos nesta estratégia na Austrália e Alemanha, quando a dominância do mercado e o criterioso planejamento de coordenação da malha surgem como fatores fundamentais para o sucesso desta estratégia competitiva (Gillen e Gados, 2008).

\subsection{Alianças (Compartilhamento de Voos)}

Por fim, pode-se citar as alianças como estratégia competitiva de resposta aos operadores de baixo custo, estratégia que ainda é pouco explorada na literatura acadêmica, mas vem recebendo maior destaque nos últimos anos.
As alianças estratégicas podem ser estruturadas em diferentes formatos. A Figura 1 mostra os principais tipos de codeshare:

- Codeshare Tradicional: Quando um itinerário com dois segmentos é comercializado por uma das companhias, mas cada empresa aérea opera um dos segmentos comercializados.

- Codeshare Virtual: Quando um itinerário com dois segmentos é comercializado por uma companhia, mas a operação dos dois segmentos é realizada pela outra companhia parceira.

De acordo com Ito e Lee (2007), a maioria dos acordos de compartilhamento de voos em nível doméstico nos Estados Unidos é considerada codeshare virtual e apresenta preços mais baixos em relação aos demais tipos de codeshare, possivelmente para capturar passageiros mais sensíveis ao preço. Entretanto, de acordo com Gayle (2012), o codeshare tradicional é mais efetivo que o virtual para combater a entrada de novos competidores uma vez que possibilita reduções reais na estrutura de custos, que permitiriam reduções mais perceptíveis no preço médio das passagens e fortaleceria a posição competitiva das empresas incumbentes no mercado além de criar uma possível barreira à entrada para novos entrantes.

Segundo Lin (2008), dependendo do cenário considerado, a formação de alianças estratégicas para deter a entrada de novos competidores tende a reduzir lucratividade das incumbentes, mas dependendo da quantidade de rotas e destinos, além do grau de diferenciação entre os produtos oferecidos no mercado em questão, a empresa incumbente pode até aumentar sua lucratividade.

O codeshare pode ser adotado também como estratégia para combater uma entrada potencial de uma Low Cost Carrier em rotas de atuação das incumbentes. Goetz e Shapiro (2012) são os primeiros autores a tratar desse tema na literatura, utilizando um modelo de probabilidade de praticar a aliança contra o operador de baixo custo, dada a ameaça de entrada do mesmo. Por meio de uma metodologia econométrica com dados desagregados em painel no nível de rotas com controle por efeitos fixos, os autores identificam que as empresas incumbentes conseguem formar alianças estratégicas com o intuito de reduzir de modo sustentável o preço médio das passagens com o propósito de mitigar uma entrada potencial de novos competidores em suas rotas de atuação, principalmente quando o poder de mercado é significativamente alto.

Nos Estados Unidos, a aliança entre concorrentes (Delta, Continental e Northwest) com rotas sobrepostas mesmo caso de Varig e TAM - permitiu ganhos de eficiência na estrutura de custos advindos da integração das malhas de rotas possibilitando a redução do preço das passagens 


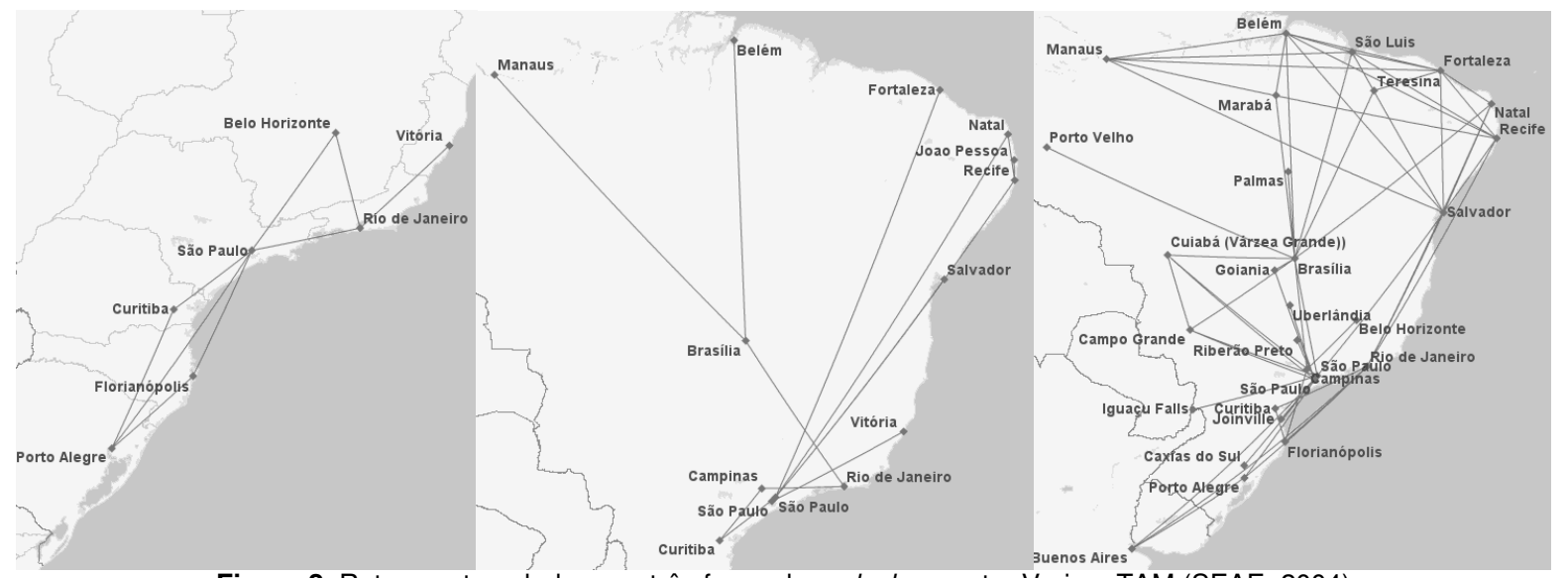

Figura 2. Rotas contempladas nas três fases do codeshare entre Varig e TAM (SEAE, 2004)

(Gayle, 2007). Em outro estudo, com a constituição do codeshare entre Continental e Northwest, Armantier e Richard (2008) concluem que o preço médio das passagens após o codeshare ficou 4,4\% abaixo da média do mercado.

\subsubsection{Benefícios das Alianças Estratégicas}

Atualmente distintos segmentos da economia tem buscado novas estratégicas para expansão ou defesa do seu negócio, as empresas tem buscado ações para unir de esforços empresariais, seja com parceiros ou concorrentes, dentre elas: acordos comerciais, alianças, fusões e aquisições. O setor de transporte aéreo não configura exceção, e as estratégias de expansão e defesa são cada vez mais exploradas. Sobre a indústria de transporte aéreo, Costa (2010) elenca as seguintes estratégias:

- Acordos Comerciais: estratégia de cooperação entre as partes, por exemplo, compartilhamento de voos.

- Aliança: sinergia da malha de rotas a fim de reduzir custos e/ou ampliar escopo dos serviços ofertados ao consumidor.

- Joint-Venture: parceria de risco entre empresas para elaboração de projetos específicos de modo independente da estrutura das partes envolvidas.

- Fusão: união total entre as empresas constituindo uma única empresa de gestão integrada, podendo uma das partes ser predominantes ou não.

- Aquisição: compra e absorção de uma empresa por outra, ou parte do negócio.

Segundo Yergin et al (2000), as alianças estratégicas tem contribuído no processo de globalização da economia e foram determinantes para expansão do transporte aéreo. Em alguns casos, estes acordos visam o fortalecimento de uma companhia em situação financeira frágil. De fato, de acordo com Andrade (2007), no ano de 2003 a Varig estava em uma grave crise quando assinou um protocolo de intenções para constituição do codeshare com a TAM. É importante mencionar que este acordo previa uma fusão total constituindo uma nova empresa. Com isso, Vassallo (2010) desenvolveu uma simulação considerando uma potencial fusão e constatou que haveria uma tendência de elevação no preço das passagens.

Através da sinergia gerada pelos acordos de codeshare, as empresas envolvidas podem obter redução dos custos, conseguir expandir sua malha aérea e disponibilizar mais opções aos passageiros. Bamberger, Carlton e Neumann (2004) afirmam que alianças proporcionam reduções de custos entre os parceiros, uma vez que ambos podem compartilhar determinadas estruturas nos aeroportos em que atuam, como lounges, posições para check-in, ações de divulgação e promoção. Os autores encontram evidências de que as empresas aéreas participantes de alianças puderam reduzir o preço entre $5 \%$ a $7 \%$.

Segundo Silveira (2007), é possível citar diversas vantagens que as alianças podem proporcionar aos passageiros, como o melhor acesso aos mercados, programas de fidelidade, dentre outros. Já para as companhias aéreas, o aumento da lucratividade com incremento de receita e o fortalecimento da posição competitiva com melhor abrangência da malha de rotas e da qualidade dos produtos ofertados aos passageiros são os principais benefícios das alianças. $\mathrm{Na}$ maioria dos casos, os acordos de codeshare resultam em economias para as companhias aéreas através da redução dos custos indiretos que são repassados na precificação das passagens aéreas, principalmente nos voos em conexão que são mais favorecidos com o codeshare. Por outro lado, para os voos diretos tende a ocorrer o oposto, por fatores competitivos, existe tendência de aumento dos preços. De acordo com Armantier e Richard (2008), estudo dedicado ao impacto da aliança doméstica entre Continental e Northwest, as alianças tem proporcionado efeitos mistos aos passageiros, o estudo encontra evidências de aumento na quantidade de passageiros em voos com conexão devido à redução do preço pago nestes voos, mas aumento do preço dos voos diretos, onde a proporção de passageiros em voos direitos reduziu.

\section{ESTUDO DE CASO: CODESHARE VARIG-TAM}

Na primeira metade da última década, no ano de 2003, a indústria de transporte aéreo no Brasil passava por um momento de grande fragilidade financeira, principalmente por parte da principal companhia aérea do país, a Varig Linhas Aéreas, que enfrentava dificuldades para honrar compromissos com credores (Andrade, 2007). Ainda em 2003, de acordo com a Secretaria de Acompanhamento Econômico (SEAE, 2004), Varig e TAM assinaram um protocolo de intenções manifestando o interesse em buscar uma solução conjunta para reestruturar o setor aéreo no Brasil, eliminando o excesso de sobreposição de voos para reduzir as perdas financeiras das empresas.

Este acordo vislumbrava inicialmente o compartilhamento recíproco de assentos nos voos (codeshare) que seriam estruturados em três fases, conforme Figura 2, abrangendo, sobretudo as rotas sobrepostas. Por fim, objetivava 
a fusão total e a constituição de uma nova empresa de capital aberto e controle privado. É possível citar dois motivos principais que justificam o modelo de codeshare por fases. Primeiro, por questão concorrencial, segundo a ANAC (2004), ambas as empresas detinham 70,7\% de participação de mercado a nível doméstico no início de 2003, sendo $37,8 \%$ da Varig e $32,9 \%$ da TAM. E finalmente, a implantação faseada permitiu que fosse possível ocorrer em paralelo, desde que a condição de reversibilidade fosse respeitada, enquanto o CADE avaliasse a situação e tivesse um parecer final.

O CADE autorizou o compartilhamento de oferta (codeshare) para permitir provisoriamente que Varig e TAM continuassem operando e não causassem maiores danos aos consumidores, mas ambas as empresas deveriam estabelecer suas estratégias comerciais de modo independente. Em adição, foi assinado um Acordo de Preservação de Reversibilidade de Operação (APRO), para garantir a reversibilidade da operação, caso o acordo fosse desfeito.

Após mais de um ano de vigente do acordo entre Varig, TAM, ficou nítido que as dificuldades financeiras enfrentadas pela Varig tinham dimensões muito maiores que na TAM, que já havia demonstrado não ter interesse em avançar com o acordo para concretizar a fusão total das companhias aéreas (Andrade, 2007). Pouco tempo depois, a SEAE (2004), através da divulgação pública da Nota Técnica n. ${ }^{\circ}$ 29/2004/COGDC-DF/SEAE/MF, identificou diversos indícios de conduta concertada de competição, inclusive com ações de redução coordenada de voos lucrativos após a efetivação do acordo de codeshare, caracterizando assim, uma hipótese de formação de cartel para aumento arbitrário da margem de lucro das empresas, política capaz de lesar os passageiros. Após investigar a racionalidade econômica da conduta, a SEAE identificou evidências de condição anticompetitiva, com indícios de exercício do poder de mercado estabelecido após o início do acordo de codeshare para extrair o maior retorno financeiro possível para as empresas mesmo que estejam prejudicando os passageiros. Dado o exposto, a SEAE recomendou ao CADE que revogasse o acordo entre as duas partes (Varig e TAM), devido aos indícios de práticas de cartel na coordenação da estratégia comercial de oferta das rotas e da precificação dos voos compartilhados pelo acordo firmado entre as empresas.

Já a Gol, que compõem a outra parte da análise, apresentava um ritmo de expansão interessante, pois conseguia introduzir rapidamente suas operações em todo o território nacional a preços mais acessíveis, se beneficiava da fragilidade financeira dos demais operadores da época e continuava a ocupar espaços deixados por Transbrasil e Vasp, que já não operaram em condições competitivas.

\section{METODOLOGIA}

Este trabalho é um estudo setorial sobre estratégias competitivas no transporte aéreo com elaboração de um modelo econométrico com efeitos fixos e controle de heteroscedasticidade para investigar as reações das empresas incumbentes à entrada de um novo competidor Low Cost Carrier por meio da implementação de um acordo de codeshare entre as empresas incumbentes, conforme o estudo de caso entre Codeshare Varig-TAM.

\subsection{Base de Dados}

Os dados utilizados foram disponibilizados pelo Departamento de Aviação Civil (DAC), órgão regulador do transporte aéreo no Brasil até 2005, quando foi substituído pela ANAC (Agência Nacional de Aviação Civil). Outras informações coletadas junto à Secretaria de Acompanhamento Econômico (SEAE) referente ao codeshare entre Varig e TAM foram inseridas à base primária de dados para que fosse possível gerar conhecimento suficiente para a análise proposta. A base de dados em painel contempla 86 rotas (sendo denominada como uma ligação aérea direcional entre duas cidades, e não aeroportos), 3 companhias aéreas incumbentes (TAM, Varig e Vasp) e 31 meses de operação (Setembro/2001 a Março/2004). Lembrando que o acordo entre Varig e TAM esteve vigente entre Março/2003 e Maio/2005.

A base de dados em painel foi compilada com dados desagregados em três níveis: a) rotas, considerando ligações aéreas entre pares de cidades, não aeroportos, b) incumbentes, companhias aéreas já estabelecidas na rota e, c) tempo, considerando os meses de operação. Decorrente da necessidade de explicitar as condições de análise do estudo de caso, foram também criadas algumas variáveis do tipo binária (dummy) para diferenciar as rotas que registraram compartilhamento de voos (codeshare) entre Varig e TAM, e a partir de qual mês isto ocorreu em cada rota analisada. Analogamente, a mesma metodologia foi aplicada para identificar as rotas em que o novo entrante Low Cost Carrier se tornou um competidor ativo e a partir de qual mês isto ocorreu em cada rota analisada.

\subsection{Definição das Variáveis}

De modo geral, estudos relacionados com análise de estratégias competitivas de resposta para combater novos entrantes tendem a empregar variável preço com dependente, já para as variáveis explicativas foi gerada a Tabela 1 com possíveis variáveis a serem empregadas no modelo econométrico proposto.

No entanto, não foi possível obter um modelo totalmente relacionado com a Tabela 1 , pois nem todas as variáveis puderam ser estruturadas e algumas variáveis não passaram no teste de hipótese e foram descartadas. Ao não usar alguns desses indicadores, a sua variabilidade ficará implícita no termo de erro não observável. Espera-se, entretanto, que o uso dos efeitos fixos possa controlar parte desses fatores não observáveis, ficando para estudos futuros a incorporação de variáveis proxy para esses fenômenos.

Para melhor entendimento da modelagem em âmbito global, optou-se por normatizar as variáveis em medidas internacionais, sendo as variáveis monetárias convertidas para dólares com valor constante de janeiro de 2010 (início da década). Em adição, os deslocadores de custos foram ponderados por frequência de voos e tipo de aeronave na operação de cada companhia aérea, assim como o nível de competição na rota. Os eventos mencionados na coluna "Especificidades Temporais" da Tabela 1 - Entrada da Low Cost Carrier na rota e Existência de Codeshare na rota são partes destacadas neste estudo, principalmente quando analisada simultaneamente a combinação dos eventos. Para isso, foram criadas combinações de dummies para registrar a ocorrência destes eventos. 
Tabela 1. Possíveis variáveis para teste na modelagem econométrica (Autores,2014)

\begin{tabular}{llll}
\hline & Condicionadores de & & \\
Deslocadores de Custo & Demanda & Indicadores de Competição & Especificidades Temporais \\
\hline Custos Operacionais & Passageiros Transportados & Incumbentes na Rota & Entrada da Low Cost Carrier na rota \\
Consumo de Combustível & Passageiro-Km Transportado & Índice de Concentração (HHI) & Existência de Codeshare na rota \\
Preço do Combustível & Carga Paga Transportada & Participação de Mercado & Combinação de ambos os casos \\
\hline
\end{tabular}

A primeira sequência dummy controla a existência do codeshare na rota, sendo a variável csh1 ou csh0, representando respectivamente a existência do codeshare ou não. Do mesmo modo, a segunda sequência dummy controla a presença do novo entrante Low Cost Carrier, sendo lcc1 ou lcc0, identificando respectivamente a presença ou não da Low Cost Carrier na rota. Como a contribuição central do presente estudo é analisar os efeitos decorrentes da combinação de ambos os fatores, ou seja, a entrada da Low Cost Carrier em rotas que possuem ou não acordos de codeshare por parte dos incumbentes, combina-se estas variáveis dummies criando três variações possíveis para a modelagem proposta, conforme Tabela 2.

Por fim, como o problema exposto em modelagens econométricas que utilizam o preço das passagens como variável dependente tendem a ser complexos devido ao número de variáveis não observáveis, fez-se necessário o emprego de um método de modelagem mais avançado com auxílio de efeitos fixos para obter ganhos de robustez na modelagem.

\subsection{Efeitos Fixos e Controle de Heteroscedasticidade}

Em econométrica, efeitos fixos são variáveis binárias atribuídas a unidades de observação que controlam as variáveis omitidas na modelagem que automaticamente recairiam sobre os resíduos do modelo. Deve-se ainda comentar que a abordagem clássica neste tipo de modelo de regressão na econometria requer outro importante controle adicional de heteroscedasticidade a fim de tratar a variância dos resíduos da equação de estimação de garantindo maiores ganhos na robustez do modelo.

O Estimador de Efeitos Fixos (Fixed Effects Estimator) é uma técnica de estimação para controlar a omissão de variáveis relevantes que se constitui em um problema de especificação do modelo com potencialidade de estimativas viesadas, em especial na circunstância em que os efeitos não observáveis sejam correlacionados com os regressores. Com o uso dos efeitos fixos, é possível incorporar na regressão a média desses elementos não observáveis e assim evitar boa parte dos problemas de estimação. Um dos modelos mais utilizados nesse sentido é o Modelo de Mínimos Quadrados com Variáveis Dummy (Least Squares Dummy Variables), que equivale ao Estimador de Efeitos Fixos (Wooldridge, 2012). Nesse caso, uma parte do esforço de modelagem recai sobre a necessidade de incorporar variáveis binárias (dummy) representativas da estrutura de painel de dados sob consideração.

Com a adição do controle por efeitos fixos algumas características de demanda, custos e operações são controladas, resultando na elaboração de variáveis dummies em três níveis desagregados e distintos: a) rota (índice $r$ ), b) incumbente (índice $i$ ), e c) tempo (índice $t$ ), sendo que as três dimensões podem ser combinadas entre si. Assume-se que cada companhia aérea incumbente pode reagir com magnitudes diferentes em cada rota e em cada do período de operação dependendo das especificidades, no entanto, os parâmetros de resposta são considerados constantes ao longo do tempo. Em resumo, pode-se pensar que o controle por efeitos fixos atua como uma alternativa para melhor modelar o impacto das variáveis omitidas da modelagem. Assumindo assim, que os principais termos omitidos (incorporados em princípio ao termo de erro aleatório) variam de acordo com a rota, incumbente e mês da operação, mas são constantes ao longo do tempo.

Como forma de exemplificar a importância dos efeitos fixos, pode-se pensar no posicionamento médio de mercado da empresa com relação ao consumidor. Cada empresa constrói, ao longo dos anos, uma imagem junto aos passageiros cujo efeito médio repassado aos preços pode ser controlado pelos efeitos fixos. Em nossa especificação, esse efeito reputacional médio da empresa pode variar ao longo do tempo (índice it, como será explicado adiante). Sem os efeitos fixos, esse fenômeno ficaria para o termo de resíduos.

Uma característica adicional da modelagem econométrica efetuada no presente estudo foi o controle de heteroscedasticidade que atua na variância do termo de erro aleatório (resíduos) da modelagem. A heteroscedasticidade também pode ser vista como resultado da ocorrência de $o u$ tliers nos dados da amostra, que significa a presença de observações com comportamento discrepante das demais observações, podendo gerar alterações significativas na regressão caso não seja tratada adequadamente (Gujarati, 2004). Neste trabalho, com o intuito de controlar a heteroscedasticidade, aplicou-se em todas as regressões, assim como a metodologia de Goolsbeee e Syverson (2008), pesos em cada observação, ponderando pela densidade da demanda para cada uma das observações contidas na base de dados.

Tabela 2. Dummies de eventos combinados para modelagem (Autores, 2014)

\begin{tabular}{lll}
\hline Variável Combinada & $\begin{array}{l}\text { Entrada da Low Cost Car- } \\
\text { rier (llc) }\end{array}$ & $\begin{array}{l}\text { Existência de Codeshare } \\
(\text { csh })\end{array}$ \\
\hline$l c c_{1} c s h_{0}$ & Sim (1) & Não (0) \\
$l c c_{0} c s h_{1}$ & Não (0) & Sim (1) \\
$l c c_{1} c s h_{1}$ & Sim (1) & Sim (1) \\
\hline
\end{tabular}




\subsection{Modelagem e Equações}

Após testes preliminares, chegou-se a uma sequência de modelagens que capturam a ideia central do estudo, ou seja, identificam influência da entrada de um operador Low-Cost quando o acordo de codeshare entre companhias incumbentes estava ou não estabelecido nas rotas, conforme o estudo de caso exposto. Para melhor entendimento da evolução da pesquisa, serão apresentados os três modelos na sequência em que foram concebidos. Todas as variáveis apresentadas nas modelagens foram analisadas considerando os três níveis de efeitos fixos: rota (índice r), incumbentes (índice i) e tempo (índice t).

Na Equação (1), apresenta-se o modelo de estimação preliminar considerando a utilização de efeitos fixos na modelagem somente no nível de rota e incumbente $\left(\gamma_{r i}\right)$ acrescido do controle adicional de tempo $\left(\gamma_{t}\right)$, representada por:

$$
\begin{aligned}
\ln \text { yield } & =\beta_{1} \ln \text { fuel }+\beta_{2} g p m+\beta_{3} \text { ninc } c_{2}+\beta_{4} \text { ninc } \\
& +\beta_{5} l c c_{1} c s h_{0}+\beta_{6} l c c_{0} c s h_{1}+\beta_{7} l c c_{1} c s h_{1}+\gamma_{r i}+\gamma_{t}+u
\end{aligned}
$$

Onde, a variável dependente ln yield representa o preço médio por milha, em logaritmo. Já o deslocador de custo ln fuel representa o custo médio do combustível por milha, em logaritmo, enquanto gpm representa o preço médio do galão de combustível por milha. Inicialmente os deslocadores de custo não passaram no teste de hipótese durante o processo de estimação, o que poderia ser explicado pela colinearidade. Deste modo, aplicou-se uma alternativa de tratamento de colinearidade sugerida por Gujarati (2004), transformando uma das variáveis em logaritmo ( $\ln$ fuel ), o que afastou a autocorrelação com a variável gpm .

Para variáveis de competição, temos ninc 2 que é uma variável binária (dummy) que registra a presença de duas empresas incumbentes na rota e ninc 3 que também é uma dummy e registra a presença de três companhias aéreas na rota. Por fim, são empregadas as variáveis dummies combinadas que são pontos de destaque da modelagem. A variável $l c c_{1} c s h_{0}$ é a dummy que registra a entrada do competidor Low Cost Carrier em rotas em que não existe o codeshare entre as incumbentes, já $l c c_{0} c s h_{1}$ é a dummy que identifica que a Low Cost Carrier não entrou nas rotas em que já existia o codeshare estabelecido entre as incumbentes. Finalmente, $l c c_{1} c s h_{1}$ é a dummy que registra a entrada da Low Cost Carrier, em rotas com a presença do codeshare entre as incumbentes. $\beta_{1}, \beta_{2}, \beta_{3}, \beta_{4}, \beta_{5}, \beta_{6}$, e $\beta_{7}$ são os coeficientes referentes às principais variáveis do modelo (além dos efeitos fixos) e $u$ representa o erro aleatório.
$\mathrm{Na}$ sequência, realizou-se o desenvolvimento da Equação (2), que apresenta as mesmas variáveis, mas além de empregar auxílio de efeitos fixos no nível de rota e incumbente $\left(\gamma_{r i}\right)$, agora também possui controle adicional por incumbentes no tempo $\left(\gamma_{i t}\right)$, sendo:

$$
\begin{aligned}
\ln \text { yield } & =\beta_{1} \ln \text { fuel }+\beta_{2} g p m+\beta_{3} \text { ninc }_{2}+\beta_{4} n i n c_{3} \\
& +\beta_{5} l c c_{1} c s h_{0}+\beta_{6} l c c_{0} c s h_{1}+\beta_{7} l c c_{1} c s h_{1}+\gamma_{r i}+\gamma_{i t}+u
\end{aligned}
$$

Finalmente, a Equação (3), representando o modelo de estimação final para esta pesquisa. Sendo uma evolução do modelo anterior com efeitos fixos no nível de rota e incumbente $\left(\gamma_{r i}\right)$ e incumbentes no tempo $\left(\gamma_{i t}\right)$. No entanto, para este modelo a variável de competição foi alterada para ninc registrando a quantidade de incumbentes na rota, a saber:

$$
\begin{aligned}
\ln \text { yield } & =\beta_{1} \ln \text { fuel }+\beta_{2} g p m+\beta_{3} \text { ninc } \\
& +\beta_{3} l c c_{1} c s h_{0}+\beta_{4} l c c_{0} c s h_{1}+\beta_{5} l c c_{1} c s h_{1}+\gamma_{r i}+\tilde{a}_{i t}+u
\end{aligned}
$$

Estas variáveis foram escolhidas, pois ln fuel e gpm exercem a função de deslocadores de custos, enquanto $n i n c_{2}, n i n c_{3}$ e ninc demonstram o nível de competição da rota. As dummies $l c c_{1} c s h_{0}, l c c_{1} c s h_{1}$ e $l c c_{0} c s h_{1}$ são variáveischave devido ao objetivo do estudo na identificação da influência conjunta dos eventos no preço médio das passagens. A Tabela 3 apresentada um sumário estatístico das variáveis utilizadas.

\section{RESULTADOS}

Considerando o desenvolvimento das três especificações matemáticas da Seção anterior, e o impacto do emprego de efeitos fixos na modelagem, elaborou-se uma análise comparativa entre os diferentes modelos e suas variáveis explicativas. A Tabela 4 apresenta os resultados estatísticos de cada modelagem - Equações (1), (2) e (3), e suas significâncias estatísticas.

Pode-se verificar que os deslocadores de custo In fuel e gpm, se mantiveram estatisticamente significantes em todas as especificações, comprovando o efeito de causalidade com a variável dependente, ln yield . Já as variáveis de competição registraram variações nas análises da significância estatística. A Equação (1) apresentou incongruência nos resultados, levando a inferir que a competição $\left(n i n c_{2}\right.$ e $\left.n i n c_{3}\right)$ teriam influência positiva, resultando em

\begin{tabular}{|c|c|c|c|c|c|c|}
\hline \multirow[b]{2}{*}{ Variável } & \multicolumn{2}{|c|}{$\begin{array}{l}l_{c c} c_{1} h_{0} \\
(O b s: 4335)\end{array}$} & \multicolumn{2}{|c|}{$\begin{array}{l}l c c_{0} c s h_{1} \\
(\text { Obs: } 1212)\end{array}$} & \multicolumn{2}{|c|}{$\begin{array}{l}l c c_{1} c s h_{1} \\
(O b s: 120)\end{array}$} \\
\hline & Média & Std. Dev. & Média & Std. Dev. & Média & Std. Dev. \\
\hline In yield & 3,9929 & 0,3722 & 4,2388 & 0,3129 & 4,8704 & 0,3447 \\
\hline ln fuel & 5,7920 & 0,2907 & 5,8010 & 0,1342 & 5,7763 & 0,0734 \\
\hline gpm & 2,3774 & 0,3873 & 2,3631 & 0,3376 & 2,3193 & 0,1180 \\
\hline $\operatorname{ninc}$ & 2,7167 & 0,5133 & 2,6584 & 0,5456 & 2,000 & 0,000 \\
\hline
\end{tabular}
aumento do preço das passagens, o que não representa a prática esperada no setor. Esta incongruência foi tratada com melhorias na especificação dos efeitos fixos na modelagem para as Equações (2) e (3). Todavia, na Equação (2)

Tabela 3. Sumário estatístico das variáveis absolutas utilizadas (Autores, 2014) 
Tabela 4. Resultados estatísticos das modelagens (Autores, 2014)

\begin{tabular}{|c|c|c|c|}
\hline Variável & $\begin{array}{l}\text { Equação (1) } \\
\gamma r i+\gamma t\end{array}$ & $\begin{array}{l}\text { Equação (2) } \\
\text { yri + yit }\end{array}$ & $\begin{array}{l}\text { Equação (3) } \\
\text { yri + yit }\end{array}$ \\
\hline \multirow[t]{2}{*}{$\ln$ fuel } & $0,0984 * * *$ & $0,0363 * *$ & $0,0362 * *$ \\
\hline & {$[0,018]$} & {$[0,016]$} & {$[0,016]$} \\
\hline \multirow[t]{2}{*}{ gpm } & $0,0796^{* * *}$ & $0,0515^{* * *}$ & $0,0515 * * *$ \\
\hline & {$[0,010]$} & {$[0,009]$} & {$[0,009]$} \\
\hline $\operatorname{ninc}$ & - & - & $\begin{array}{l}-0,0701 * * * \\
{[0,015]}\end{array}$ \\
\hline$n i n c_{2}$ & $\begin{array}{l}0,0783 * * \\
{[0,039]}\end{array}$ & $\begin{array}{l}0,0303 \\
{[0,031]}\end{array}$ & - \\
\hline $\operatorname{ninc}_{3}$ & $\begin{array}{l}0,0452 \\
{[0,044]}\end{array}$ & $\begin{array}{l}-0,0689^{*} \\
{[0,036]}\end{array}$ & - \\
\hline$l c c_{1} c s h_{0}$ & $\begin{array}{l}-0,1189 \text { *** } \\
{[0,017]}\end{array}$ & $\begin{array}{l}-0,1141 * * * \\
{[0,014]}\end{array}$ & $\begin{array}{l}-0,1236 * * * \\
{[0,013]}\end{array}$ \\
\hline$l c c_{1} c s h_{1}$ & $\begin{array}{l}-0,1041 * * * \\
{[0,021]}\end{array}$ & $\begin{array}{l}-0,0771 * * * \\
{[0,019]}\end{array}$ & $\begin{array}{l}-0,0830 * * * \\
{[0,019]}\end{array}$ \\
\hline$l c c_{0} c s h_{1}$ & $\begin{array}{l}0,0301 \\
{[0,039]} \\
\end{array}$ & $\begin{array}{l}0,0134 \\
{[0,032]}\end{array}$ & $\begin{array}{l}0,0169 \\
{[0,032]}\end{array}$ \\
\hline Notas: & $\begin{array}{l}\text { Ses estimados } \\
\text { ções de p-valo } \\
\text { inalisadas con }\end{array}$ & $\begin{array}{l}* * \mathrm{p}<0,05 \text { e } \mathrm{p}< \\
\mathrm{s} \text { fixos em } 3 \mathrm{n}\end{array}$ & ncumbentes (i) e tempo (t). \\
\hline
\end{tabular}

apesar de ter corrigido o viés de correlação e passar no teste de significância a 10\%, não foi tão satisfatória. Nota-se que nas Equações (1) e (2), as variáveis referentes ao nível de competição não apresentaram os resultados esperados, o que poderia demandar melhor atenção em eventuais estudos futuros.

Como solução para este estudo, optou-se em incorporar a variável ninc (quantidade de incumbentes na rota) na Equação (3) como indicador de competição, pois registrou a correlação negativa esperada com significância estatística de $1 \%$.

As variáveis de evento (dummies) registraram o comportamento esperado em todos os casos demonstrando relações de causalidade coerentes com a realidade do transporte aéreo e com significância estatística de 1\%. De fato, nos dois cenários de entrada da Low Cost Carrier na rota ( $l c c_{1} c s h_{0}$ e $l c c_{1} c s h_{1}$ ), ocorreram reduções nos preços das passagens conforme esperado e já amparado na literatura acadêmica, pois tipicamente este modelo de negócio pratica precificação abaixo da média praticada pelas empresas incumbentes.

Quando o competidor Low Cost entrou na rota, mas não estava estabelecido o codeshare pelas incumbentes $\left(l c c_{1} c s h_{0}\right)$, a redução dos preços foi $12,36 \%$, em média. Por outro lado, quando o codeshare já estava implementado ( $l c c_{1} c s h_{1}$ ), a redução dos preços com a entrada da Low Cost Carrier na rota foi apenas 8,30\%, em média. Deste modo, pode-se inferir que há evidências de que a estratégia de $c o$ deshare contempla a possibilidade de suavizar a ação de redução de preço das passagens advindas da entrada do competidor Low Cost Carrier.

Esta evidência foi verificada estatisticamente através do teste de diferenças entre médias, considerando a hipótese de que os coeficientes de $l c c_{1} c s h_{0}$ e $l c c_{1} c s h_{1}$ eram iguais. Essa hipótese foi rejeitada a 1\% (P-valor igual a 0.0007) indicando que os valores de redução encontrados na modelagem final (Equação 3) são estatisticamente diferentes entre si.

Já no caso da variável $l c c_{0} c s h_{1}$ que identifica a nãoentrada do competidor Low Cost em rotas que o codeshare entre Varig e TAM estava ativo, não apresentou significância estatística, o que demonstra que as empresas incumbentes não conseguiram modificar consideravelmente a estrutura de precificação em rotas com menor pressão competitiva. Este resultado é relevante, pois um acréscimo na precificação de mercados menos competitivos culminaria na exposição ao risco, seja pela eventual perda de demanda ou pela exposição ao interesse de potenciais novos competidores, sejam estes Low Cost Carriers ou não, uma vez que esta rota passaria a registrar margens de lucro mais atrativas.

\section{CONCLUSÃO}

O presente trabalho buscou efetuar um estudo setorial sobre estratégias competitivas no transporte aéreo e o papel da formação de alianças entre companhias aéreas. Teve como principal objetivo avaliar a relação competitiva do tipo de aliança estratégia conhecido no setor como "codesharing" (acordo de compartilhamento de voo) através dos efeitos gerados com a implementação do acordo entre empresas incumbentes frente à entrada de um competidor de operação de baixo custo (Low Cost Carrier). Este tema tem sido pouco explorado na literatura acadêmica, e por isso constitui a contribuição temática deste trabalho.

Baseada na literatura existente elaborou-se uma metodologia através do desenvolvimento de uma modelagem econométrica com auxílio de efeitos fixos em três níveis i) rota (pares de cidades), ii) incumbente (empresa aérea) e iii) tempo (meses) - e controle de heteroscedasticidade a fim de tratar as variáveis omitidas do modelo e a variância dos resíduos da equação de estimação de garantindo maiores ganhos na robustez do modelo.

Em termos metodológicos, a principal contribuição do estudo é o desenvolvimento da modelagem com variáveis binárias (dummies) que avaliam simultaneamente os efeitos combinados das reações no preço médio das passagens após entrada do competidor Low Cost Carrier nas rotas em que o acordo de codeshare entre empresas incumbentes já estava em vigor ou não.

Conforme esperado, os resultados indicam reduções dos preços quando a Low Cost Carrier entra na rota. Nas condições sem constituição do codeshare, a redução foi 
$12,36 \%$, em média. E quando o codeshare já estava em vigor, a redução foi de apenas $8,30 \%$, em média. Estes resultados foram validados estatisticamente por teste de hipóteses das diferenças entre as estimativas, e rejeitou-se a igualdade no padrão de reações com significância de $1 \%$. Esta análise estatística possibilita inferir que há evidências de que a estratégia de codeshare, apesar de não neutralizar, pode ser uma alternativa para suavizar a ação de redução de preços das passagens advindas da competição direta com Low Cost Carrier.

Analogamente, foi replicado o mesmo procedimento para casos em que a Low Cost Carrier não entrou, mas as empresas incumbentes implementaram o codeshare. Este caso não apresentou significância estatística conclusiva, o que sugere que as companhias incumbentes não conseguiram modificar representativamente a estrutura de precificação das passagens em rotas com menor pressão competitiva, pois culminaria na exposição deste mercado ao risco de entrada de potenciais competidores, sejam estes Low Cost Carriers ou não.

\section{REFERÊNCIAS}

ANAC (2003). Anuários Estatísticos do Transporte Aéreo - Dados Estatísticos. Disponível em: http://www2.anac.gov.br/estatis tica/anuarios.asp

ANDRADE, T. A. (2007). A crise Varig/TAM e o uso de codeshare. Regulação no Brasil, Instituto de Pesquisa Econômica Aplicada, 165-173.

ARMANTIER, O. e RICHARD, O. (2008). Domestic airline alliances and consumer welfare. RAND Journal of Economics, v. 39, n. 3, p. 875-904. DOI: 10.1111/j.1756-2171.2008.00042.x.

BAMBERGER, G. E., CARLTON, D. W., e NEUMANN, L. R. (2004). An empirical investigation of the competitive effects of domestic airline alliances. The Journal of Law Economics, v. 47, n. 2, p. 195-222. DOI: $10.1086 / 386274$.

COSTA, W. C. (2010). Alianças domésticas entre companhias aéreas e o impacto no comportamento dos passageiros. Journal of Transport Literature, v. 4, n. 1, p. 96-113.

DOGANIS, R. (2001). Flying of Course: The Economics of International Airlines. London: Routledge, $2^{a}$ Edição.

GATIGNON, H., ROBERTSON, T., e FEIN, A. J. (1997). Incumbent defense strategies against new product entry. International Journal of Research in Marketing 14(2), 163-176. DOI: 10.1016/S0167-8116(96)00035-3.

GAYLE, P. G. (2007). Airline code-share alliances and their competitive effects. Journal of Law and Economics 50, 781-819.

GAYLE, P. G., e XIE, X. (2012). Entry deterrence and strategic alliances: evidence from a dynamic structural econometric model. Working paper.

GILLEN, D., e GADOS, A. (2008). Airlines within airlines: Assessing the vulnerabilities of mixing business models. Research in Transportation Economics 24, 25-35. DOI: 10.1016/j.retrec. 2009.01.002.

GOETZ, C. F., e SHAPIRO, A. H. (2012). Strategic alliance as a response to the threat of entry: Evidence from airline codesharing.
International Journal of Industrial Organization 30, 735-747. DOI: 10.1016/j.ijindorg.2012.08.003.

GOOLSBEE, A., e SYVERSON, C. (2008). How do incumbents respond to the threat of entry? Evidence from the major airlines. Quarterly Journal of Economics, 123, 1611-1633. DOI: 10.1162/qjec.2008.123.4.1611.

GUJARATI, D. (2004). Basic Econometrics, $4^{\text {th }}$ edition. The McGraw-Hill Companies.

HÜSCHELRATH, K., e MÜLLER, K. (2011). Low cost carriers and the evolution of the U.S. airline industry. ZEW - Centre for European Economic Research Discussion No. 11-051. DOI: 10.2139/ssrn. 1908744 .

HUSE, C., e OLIVEIRA, A. V. M. (2012). Does product differentiation soften price reactions to entry? Evidence from the airline industry. Journal of Transport Economics and Policy 46, Part 2, 01-16. DOI: 10.2139/ssrn.1709070.

ITO, H., e LEE, D. (2007). Domestic codesharing, alliances and airfares in the U.S. airline industry. Journal of Law \& Economics, 50, 355-380. DOI:10.1086/511318.

LIN, M. H. (2008). Airline alliances and entry deterrence. Transportation Research Part E 44, 637-652. DOI: 10.1016/j.tre.2007.05.003.

MORRELL, P. (2005). Airlines within airlines: An analysis of US network airline responses to low cost carriers. Journal of Air Transport Management 11, 303-312. DOI: 10.1016/j.jairtraman. 2005.07.002.

OLIVEIRA, A. V. M. (2008). An empirical model of low-cost carrier entry. Transportation Research Part A 42, 673-695. DOI: 10.1016/j.tra.2008.01.025.

OLIVEIRA, A. V. M., FERREIRA, N. S. e SALGADO, L. H. (2011) A liberalização econômica do transporte aéreo no Brasil: um estudo empírico dos dez primeiros anos. Transportes 19, 6274. DOI: $10.4237 /$ transportes.v19i3.369

SEAE (2004). Secretaria de Acompanhamento Econômico - Nota Técnica n. ${ }^{\circ}$ 29/2004/COGDC-DF/SEAE/MF. Coordenação Geral de Defesa da Concorrência, Brasília.

SILVEIRA, D. M. S. (2007). Análise dos benefícios das alianças internacionais entre empresas de transporte aéreo. Dissertação Universidade Federal do Rio de Janeiro, COPPE.

TAN, K. M. (2011). Incumbent Response to Entry by Low-Cost Carriers in the U.S. Airline Industry. Working paper. DOI: $10.2139 / \mathrm{ssrn} .2006471$

VASSALlO, M. D., (2010). Simulação de Fusão com Variações de Qualidade no Produto das Firmas: Aplicação para o Caso do Code-Share Varig-TAM. Journal of Transport Literature 4, 50100.

YERGIN, D., VIETOR, R. H. K. e EVANS, P. C. (2000). Fettered Flight: Globalization and the Airline Industry. Massachussets Institute of Technology.

WINDLE, R., e DRESNER, M. (1999). Competitive responses to low cost carrier entry. Transportation Research Part E 35, 59-75.

WOOLDRIGDE, J. M. (2012). Introductory Econometrics: a modern approach. $5^{\text {th }}$ edition, South-Western Cengage Learning. 
WU, S. M. (2011). The "Southwest effect" revisited: An empirical analysis of the effects Southwest and Jetblue Airways on incumbent airlines from 1993 and 2009. The Michigan Journal of Business. 